\title{
Bioinformatics screening regarding herbal components that targetedly regulate the function of tumour-associated macrophages
}

\author{
BO GAO $^{1}$, YINGMEI WANG ${ }^{1}$, YAN QIU ${ }^{1}$, WEILIANG XIA ${ }^{1}$, XIANZHI MENG ${ }^{1}$, MING LU $^{2}$, \\ WEIHUI ZHANG ${ }^{1}$, XIN QIAO $^{2}$, YINGMEI ZHANG ${ }^{3}$ and DONGBO XUE ${ }^{1}$ \\ ${ }^{1}$ Department of General Surgery, The First Affiliated Hospital of Harbin Medical University, Harbin, \\ Heilongjiang 150001, P.R. China; ${ }^{2}$ Department of Surgery, David Geffen School of Medicine, \\ University of California at Los Angeles, Los Angeles, CA 90095, USA; ${ }^{3}$ Central Laboratory, \\ The First Affiliated Hospital, Harbin Medical University, Harbin, Heilongjiang 150001, P.R. China
}

Received February 26, 2014; Accepted April 17, 2014

DOI: $10.3892 / o r .2014 .3217$

\begin{abstract}
As an important component of tumour stroma, tumour-associated macrophages (TAMs) promote tumour development and progression. Herbs have been increasingly used in anticancer therapies due to their wide-ranging anticancer effects and minor side-effects. However, no herb-based treatments targeting TAMs have yet been proposed. To address this issue, screening using modular analysis bioinformatics techniques found 6 core functional modules for TAMs that contain 46 total genes. Moreover, 15 potential new anticancer drugs that regulate the genes in the 6 core modules were identified through bioinformatics techniques and Fisher's exact test. Our results provide a new research avenue for targeting TAMs in anticancer therapies.
\end{abstract}

\section{Introduction}

Tumour-associated macrophages (TAMs) are the most common type of inflammatory cells in tumour stroma. In previous years, TAMs were found to be closely associated with tumour formation and development $(1,2)$. Studies have shown that TAMs not only secrete cytokines, stimulating tumour cell proliferation and survival, but also produce a variety of mesenchymal regulatory proteins that promote tumour metastasis (3-5). Moreover, TAMs act on endothelial cells, making

Correspondence to: Professor Yingmei Zhang, Central Laboratory, The First Affiliated Hospital, Harbin Medical University, 23 You Zheng Street, Harbin, Heilongjiang 150001, P.R. China

E-mail: zhangyingmei1111@126.com

Professor Dongbo Xue, Department of General Surgery, The First Affiliated Hospital of Harbin Medical University, 23 You Zheng Street, Harbin, Heilongjiang 150001, P.R. China

E-mail: hmu_xuedongbo@163.com

Key words: tumour-associated macrophages, herbal components, modular analysis, anticancer therapies them a key factor in tumour angiogenesis and lymphangiogenesis (6). In vitro studies have shown that malignant cells cannot grow after TAMs are knocked out (7) and it has also been confirmed that TAM-mediated immunosuppression promotes tumour immune escape (8). This evidence demonstrates that TAMs play a role throughout tumour formation, growth and metastasis, and that they have a powerful tumour-promoting effect.

Tumours, particularly malignant tumours, are a leading cause of mortality around the world. Conventional cancer treatments include surgery, radiotherapy and chemotherapy, but the use of these treatments is greatly limited due to poor prognoses and severe side-effects. At present, traditional Chinese medicine, which is an aspect of traditional Chinese culture, is seeing increased use as a cancer treatment method. In particular, traditional Chinese medicinal herbs are being employed as new anticancer drugs. Statistics indicate that half of the internationally recognized anticancer drugs in use from the 1940 to 2006 were either natural products or their derivatives, indicating that Chinese herbs have considerable potential as antitumour treatments $(9,10)$. However, existing studies on the anticancer effects of herbal components have largely focused on the effects of these components on solid tumour cells; few studies have focused on TAMs, which have a strong tumour-promoting effect. Given the notable anticancer effects of herbal components and the key role TAMs play in tumour growth and development, a cancer treatment using herbal components to target TAMs, either alone or in combination with traditional methods, may become a new and effective anticancer approach.

Modular analysis bioinformatics techniques (in model organisms) have become popular in disease and drug research since they are capable of not only elucidating the local features of gene regulatory networks but also conducting a comprehensive and systematic analysis of regulatory signalling pathways $(11,12)$. In the present study, we used modular analysis bioinformatics techniques to screen for the differentially expressed genes in TAMs that play a role in their core functional group(s). Using the connectivity map (cmap) 
Table I. Summary of 4 data series (GSE38283, GSE18804, GSE18404 and GSE36047).

\begin{tabular}{|c|c|c|c|c|}
\hline Series & Array platform & Samples & Experiment design & Group \\
\hline \multirow{7}{*}{$\begin{array}{l}\text { GSE38283 } \\
\text { Expression data } \\
\text { from normal } \\
\text { brain/glioma } \\
\text { associated } \\
\text { macrophages }\end{array}$} & GPL1261 & GSM937959 & Macrophages from normal brain, Rep 1 & Control \\
\hline & \multirow{6}{*}{$\begin{array}{l}\text { Affymetrix } \\
\text { mouse genome } \\
4302.0 \text { array }\end{array}$} & GSM937960 & Macrophages from normal brain, Rep 2 & Control \\
\hline & & GSM937961 & Macrophages from normal brain, Rep 3 & Control \\
\hline & & GSM937962 & Macrophages from Gl261, Rep 1 & TAM \\
\hline & & GSM937963 & Macrophages from Gl261, Rep 2 & TAM \\
\hline & & GSM937964 & Macrophages from G1261, Rep 3 & TAM \\
\hline & & GSM937965 & Macrophages from G1261, Rep 4 & TAM \\
\hline \multirow{9}{*}{$\begin{array}{l}\text { GSE18804 } \\
\text { Expression data from } \\
\text { in vitro-induced } \\
\text { non-hypoxic TAMs }\end{array}$} & \multirow{9}{*}{$\begin{array}{l}\text { GPL6246 } \\
\text { Affymetrix } \\
\text { mouse gene } 1.0 \\
\text { ST array }\end{array}$} & GSM466436 & Macrophage unconditioned, Rep 1 & Control \\
\hline & & GSM466437 & Macrophage unconditioned, Rep 2 & Control \\
\hline & & GSM466438 & Macrophage unconditoned, Rep 3 & Control \\
\hline & & GSM466439 & $\begin{array}{l}\text { TAMs_human glioblastoma-conditioned, } \\
\text { Rep } 1\end{array}$ & TAM \\
\hline & & GSM466440 & $\begin{array}{l}\text { TAMs_human glioblastoma-conditioned, } \\
\text { Rep } 2\end{array}$ & TAM \\
\hline & & GSM466441 & $\begin{array}{l}\text { TAMs_human glioblastoma-conditioned, } \\
\text { Rep } 3\end{array}$ & TAM \\
\hline & & GSM466442 & $\begin{array}{l}\text { TAMs_human colorectal adenocarcinoma- } \\
\text { conditioned, Rep } 1\end{array}$ & TAM \\
\hline & & GSM466443 & $\begin{array}{l}\text { TAMs_human colorectal adenocarcinoma- } \\
\text { conditioned, Rep } 2\end{array}$ & TAM \\
\hline & & GSM466444 & $\begin{array}{l}\text { TAMs_human colorectal adenocarcinoma- } \\
\text { conditioned, Rep } 3\end{array}$ & TAM \\
\hline \multirow{8}{*}{$\begin{array}{l}\text { GSE18404 } \\
\text { High-density gene } \\
\text { expression analysis of } \\
\text { TAMs from mouse } \\
\text { mammary tumours }\end{array}$} & \multirow{8}{*}{$\begin{array}{l}\text { GPL9298 } \\
\text { Nimbelgen } \\
\text { mouse 35K } \\
\text { array }\end{array}$} & GSM458919 & TAM, Rep 1 & TAM \\
\hline & & GSM458920 & TAM, Rep 2 & TAM \\
\hline & & GSM458921 & TAM, Rep 3 & TAM \\
\hline & & GSM458922 & TAM, Rep 4 & TAM \\
\hline & & GSM458923 & Splenic Macrophage, Rep 1 & Control \\
\hline & & GSM458924 & Splenic Macrophage, Rep 2 & Control \\
\hline & & GSM458925 & Splenic Macrophage, Rep 3 & Control \\
\hline & & GSM458926 & Splenic Macrophage, Rep 4 & Control \\
\hline GSE36047 & GPL13252 & GSM879917 & Macrophage cultured for $6 \mathrm{~h}$ & Control \\
\hline $\begin{array}{l}\text { Macrophages in } \\
\text { invadopodia }\end{array}$ & $\begin{array}{l}\text { Agilent-027114 } \\
\text { human } 8 \times 60 \mathrm{k}\end{array}$ & GSM879918 & $\begin{array}{l}\text { Macrophage cocultured with tumour cells } \\
\text { (DU-145) for } 6 \mathrm{~h}\end{array}$ & TAM \\
\hline
\end{tabular}

TAMs, tumour-associated macrophages.

small molecule drug database, we identified herbal components that regulate the genes in these core functional groups, thereby establishing a regulatory network between the herbal components and the functional gene groups. The present study provided a more in-depth analysis of the functions of TAMs and screened for a large number of herbal components that regulate them, offering a new entry point for future anticancer treatment research.

\section{Materials and methods}

Screening for differentially expressed genes in TAMs. We searched the National Center for Biotechnology Information (NCBI) PubMed database to retrieve the genome-wide expression profiles of mouse and human TAMs (GSE38283,
GSE18804, GSE18404 and GSE36047). Summary of the 4 data series is shown in Table I. Of these profiles, GSE38283, GSE18804 and GSE18404 were analyzed for the presence of differential gene expressions using the significance analysis of microarrays (SAM) approach. A false discovery rate (FDR) of $<0.1$ was considered to represent differential expression (Fig. 1). GSE36047 was screened for differentially expressed genes based on fold-changes. In addition, BioMart software was used to identify the human genes that were homologous to the differentially expressed mouse genes.

Establishing and analysing the modules of the differentially expressed genes. The Human Protein Reference Database (HPRD) was used to obtain a protein-protein interaction (PPI) network that included 36,874 edges and 9,453 nodes. The 

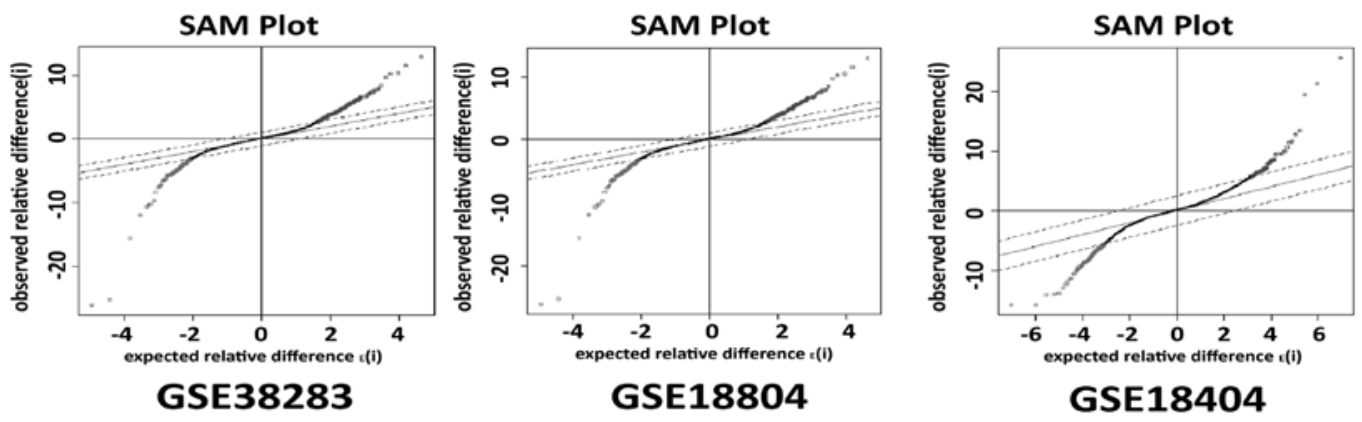

Figure 1. Screening for differentially expressed genes in TAMs. The genome-wide expression profiles of mouse and human TAM (GSE38283, GSE18804, GSE18404 and GSE36047) were retrieved from the National Center for Biotechnology Information (NCBI) PubMed database. Of these profiles, GSE38283, GSE18804 and GSE18404 were analyzed for the presence of differential gene expressions using the significance analysis of microarrays (SAM) approach. A false discovery rate (FDR) of $<0.1$ was considered to represent differential expression. TAMs, tumour-associated macrophages.

differentially expressed genes were mapped to the PPI network, and pairs of interacting genes in which both genes were differentially expressed were noted, creating sub-networks. Subsequently, CFinder software was used to identify functional modules in the sub-networks. Then, Database for Annotation, Visualization and Integrated Discovery (DAVID) software was used to screen the modules for gene ontology-biological processes (GO_BP), and the Kyoto Encyclopedia of Genes and Genomes (KEGG) was used to perform a database-based pathway enrichment analysis.

Screening for traditional Chinese medicine components that regulate the functional modules. The cmap database stores the genome-wide transcription and expression profiles of human cells that have been treated with various active small molecules. The cmap includes 6,100 small molecule interference experiments, which use both a test group treated with the small molecule and a control group, for a total of 7,056 different expression profiles. The small molecule interference experiments tested 1,309 different small molecules. Using these interference experiment results, we analyzed the expression profile of each drug to identify the differentially expressed genes by comparing drug administration to no drug administration. The genes that were differentially expressed by each drug were regarded as the gene cluster affected by the drug in question. We then mapped the genes in each screened module described above with the differentially expressed genes determined via cmap to obtain the relationships between the genes in each module and the tested small molecules. Fisher's exact test was used to statistically evaluate these relationships, and the 10 small molecules with the highest p-values were considered to have the most potential.

Literature review and the generation of a regulatory network connecting herbal components and modules. To obtain new, possibly valuable anticancer herbal components, we conducted a literature review focused on the studies of the anticancer effects of the 10 herbal components that most regulated the genes in the screened modules. First, the Perl software language was used to write a literature review program. Next, ActivePerl 5.16.2 software was used to retrieve literature data from the NCBI PubMed database. The search locations were the titles and abstracts of publications, and the search keywords were the names of the screened herbal components and the
Table II. Fisher's exact test.

\begin{tabular}{llll}
\hline & $\begin{array}{c}\text { Module } \\
\text { gene }\end{array}$ & \multicolumn{1}{c}{$\begin{array}{c}\text { No module } \\
\text { gene }\end{array}$} & \multicolumn{1}{c}{ Total } \\
\hline $\begin{array}{l}\text { In term } \\
\text { Not in }\end{array}$ & $\mathrm{m}(\mathrm{a})$ & $\mathrm{y}-\mathrm{m}(\mathrm{b})$ & $\mathrm{y}$ \\
term & $\mathrm{Y}-\mathrm{M}-\mathrm{y}+\mathrm{m}(\mathrm{d})$ & $\mathrm{Y}-\mathrm{y}$ \\
Total & $\mathrm{M}$ & $\mathrm{Y}-\mathrm{M}$ & $\mathrm{Y}(\mathrm{n}=\mathrm{a}+\mathrm{b}+\mathrm{c}+\mathrm{d})$ \\
\hline
\end{tabular}

$\mathrm{Y}$, the total number of genes; $\mathrm{M}$, the number of module genes; $\mathrm{y}$, the number of genes that are regulated by molecule $\mathrm{X} ; \mathrm{m}$, the number of module genes that are regulated by molecule $\mathrm{X}$.

word 'cancer'. Finally, we retrieved the number of publications related to each herbal component and cancer. The results of the herbal component screening and the findings from the literature review were integrated with the gene modules, and Cytoscape software was used to generate a regulatory network connecting the herbal components to the target genes.

Statistical analysis. T-tests were used to screen the differentially expressed genes and to evaluate the results of the genes' GO_BP and KEGG-based enrichment analyses. T-tests were also conducted on the gene chips that were repeated three times or more (GSE38283, GSE18804 and GSE18404). Genes with an FDR of $<0.1$ were considered to be differentially expressed. For gene chips that were repeated $<3$ times (GSE36047), the fold-change was used as the criterion, and genes with a fold-change $>2$ or $<0.5$ were considered to be differentially expressed. To evaluate the results of the GO_BP and KEGGbased enrichment analyses, $\mathrm{p}<0.01$ was considered statistically significant. Fisher's exact test was used to evaluate the genes in the modules with respect to each herbal component. The principle for this choice is shown in Table II. A p-value for each component was then calculated based on the above data, using the following equation:

$$
p=\frac{\left(\begin{array}{c}
a+b \\
a
\end{array}\right)\left(\begin{array}{c}
c+d \\
c
\end{array}\right)}{\left(\begin{array}{c}
n \\
a+c
\end{array}\right)}=\frac{(a+b) !(c+d) !(a+c) !(b+d) !}{a ! b ! c ! d ! n !}
$$




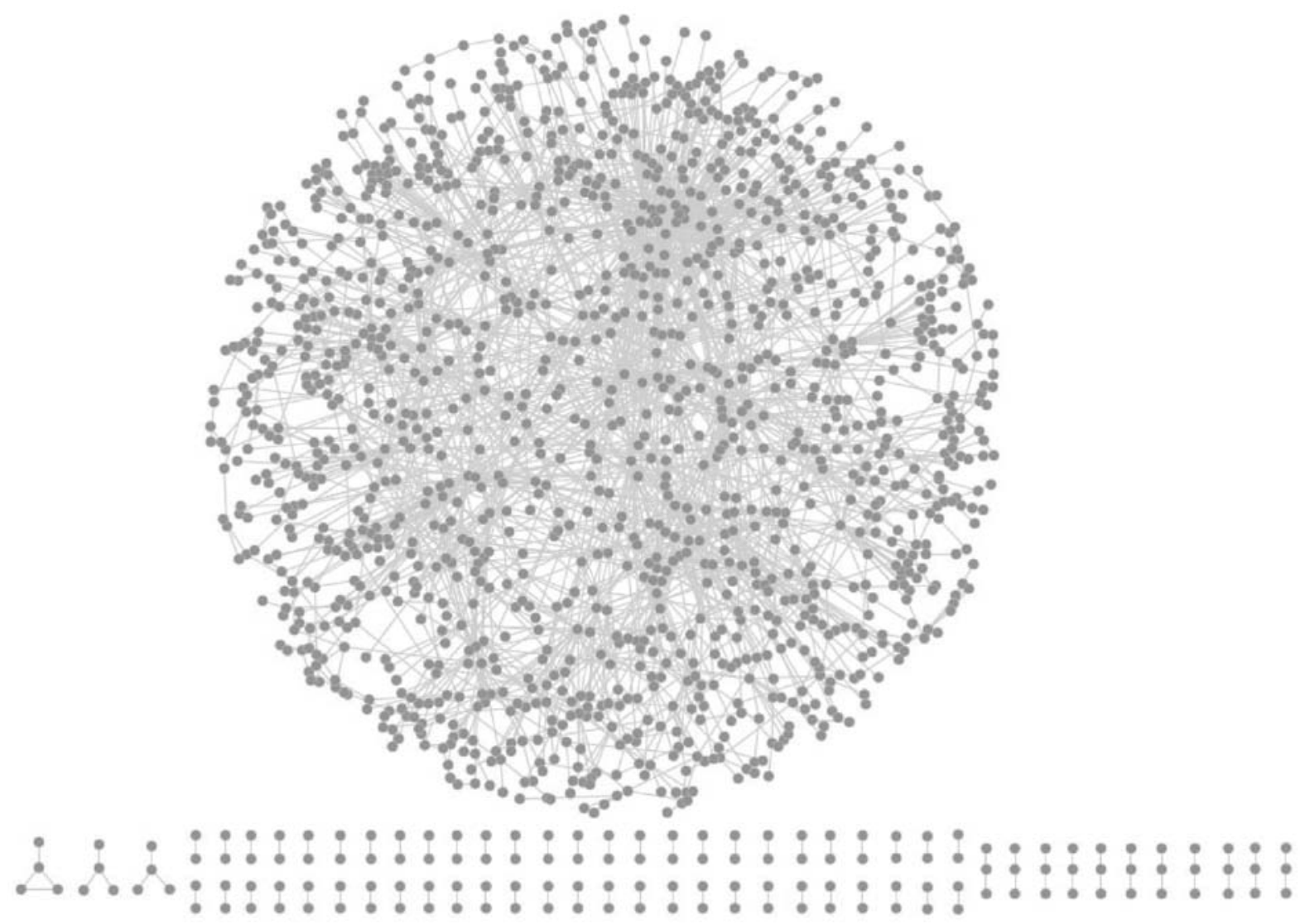

Figure 2. Sub-networks of interacting genes with differential expressions. Among the four groups of TAM expression profiles, a total of 4,753 differentially expressed genes were identified. These differentially expressed genes were mapped to the PPI network, and pairs of interacting genes in which both genes were differentially expressed were used to generate sub-networks, which had 2,407 edges and 1,501 nodes. TAMs, tumour-associated macrophages.

\section{Results}

Screening the gene modules. Among the four groups of TAM expression profiles, a total of 4,753 differentially expressed genes were identified. These differentially expressed genes were mapped to the PPI network, and pairs of interacting genes in which both genes were differentially expressed were used to generate sub-networks, which had 2,407 edges and 1,501 nodes (Fig. 2). Next, CFinder software was used to identify modules within the sub-networks. When $\mathrm{k}=4,6$ modules were identified that had a total of 46 genes. We named them modules 1-6 (Fig. 3A).

Functional analyses of the gene modules. GO_BP and KEGGbased enrichment analyses were conducted on the 6 modules. The resulting GO terms and pathways found for each module were ranked according to their enrichment scores [-log (t-test p-values)]. The top 10 GO_BP terms and KEGG pathways, which each had $p$-values of $<0.01$, were considered to be the most valuable and were used for subsequent analyses. The $\mathrm{GO}$ analysis demonstrated that the main function of module 1 was positive regulation of transcription, positive regulation of biosynthetic process and so on; the function of module 2 was angiogenesis, vasculature development and tube development; the function of module 3 was peptidyl-tyrosine phosphorylation, cell surface receptor linked signal transduction and protein kinase cascade, and the main function of modules 4 , 5 and 6 was regulation of cell cycle (Fig. 3B). Expression results from the pathway analysis showed that for modules 1 and 2, cancer-related pathways were the most significant. For module 3, the Janus kinase-signal transducers and activators of transcription (JAK-STAT) signalling pathway and the chemokine pathways were the most significant. For modules 4,5 and 6 , the cell cycle pathways were the most significant (Fig. 3C).

Herbal components that regulate the gene modules. Based on the cmap database, we calculated that genes were differentially expressed in 1,309 small molecule interference experiments, and the final results showed that 1,221 small molecules caused differential expressions. We then mapped each of the 46 genes identified in the 6 modules with the differentially expressed genes obtained from the cmap; this mapping allowed us to describe the relationships between the genes in each module and the small molecules. Specifically, we looked at the small molecules that are components of the traditional Chinese medicine herbs. Fisher's exact test and the original data were used to calculate the p-value of each herbal component for each module; the p-values were ranked in descending order, and the herbal components with the 10 smallest $p$-values were considered to have the most value and were used for subsequent analyses (Fig. 4). The result showed that a total of 44 herbal components were identified as regulators of the 6 modules.

Potential new anticancer drugs. To obtain novel anticancer drugs, we conducted a literature review of the cancer-related 


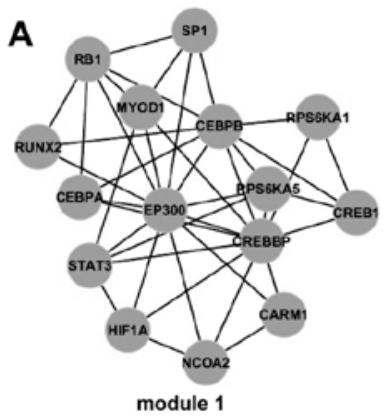

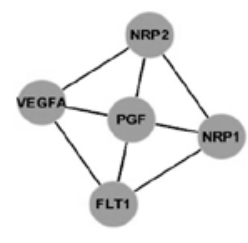

module 2
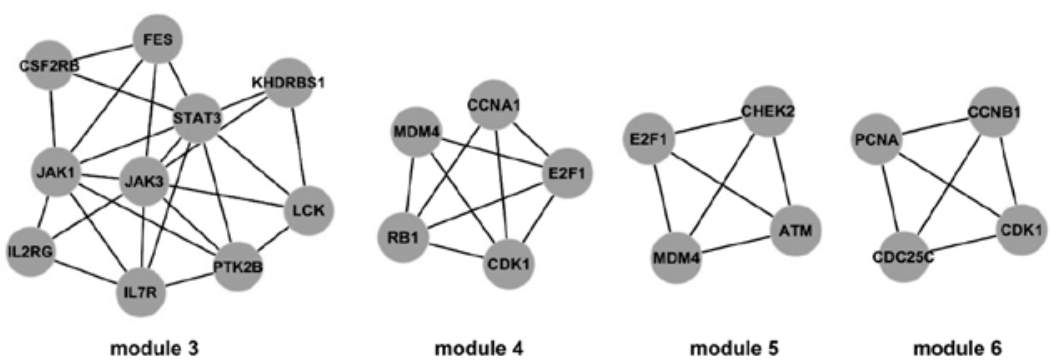

B GO analysis:

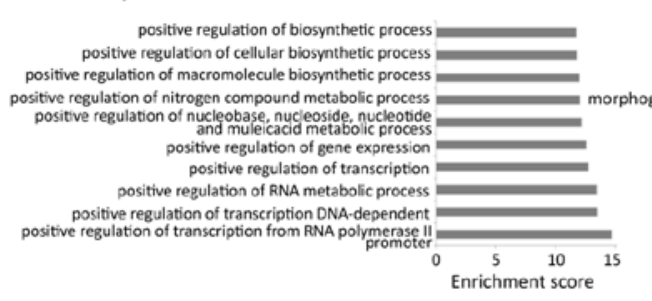

module 1

negative regulation of RNA metabolic process

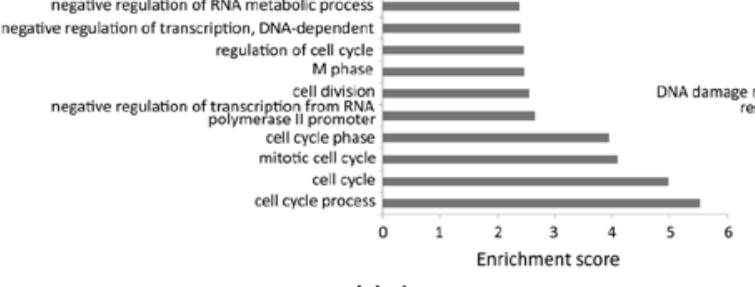

module 4

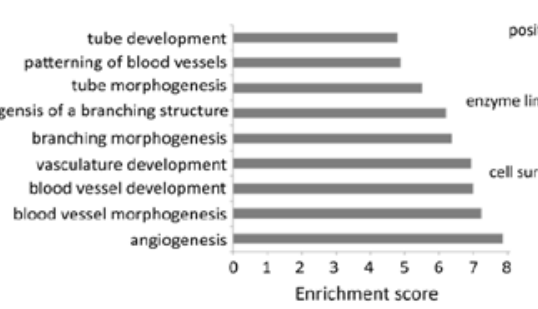

module 2

A integrity checkpoint

DNA damage checkpoint

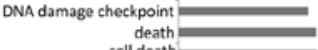

cell death

tosis

programmmed cell death
protion

apoptosis
cell cycle process

regulation of cycle
cell cycle

cell cycle

module 5

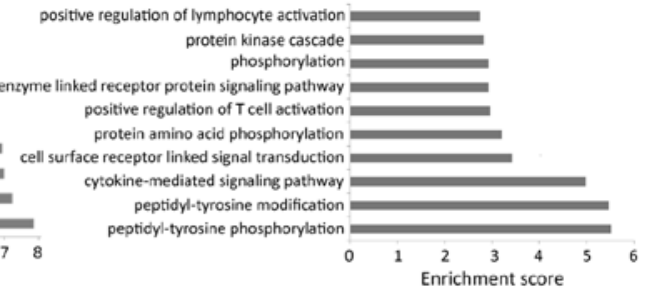

module 3

C Pathway analysis:
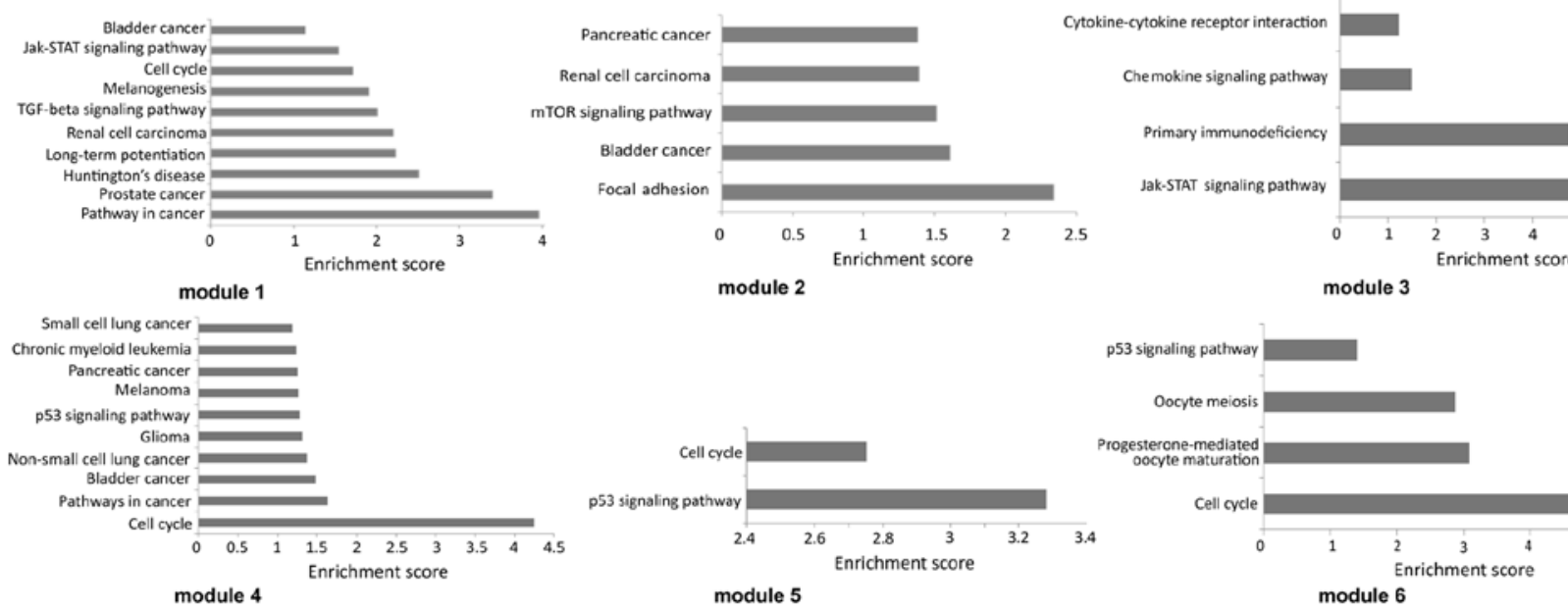

Jak-STAT signaling pathway

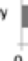

cell cycle process

cell cycle phase
mitotic cell cycle

egulation of cell cycle
M phase

M phase
cell division

organelle fission

mitotic cell cycle
mittosis
nuclear division

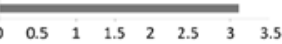

module 6 module 2

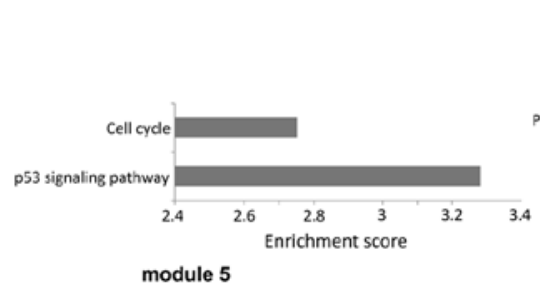

module 5

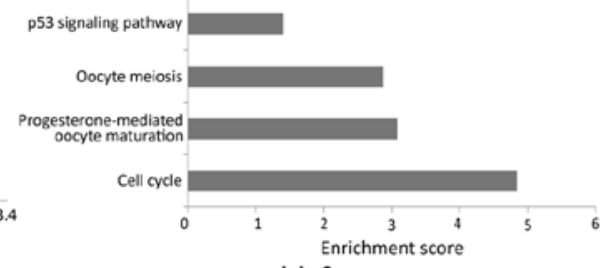

module 6

Figure 3. Screening and bioinformatics analysis of modules. (A) Sub-networks of interacting genes with differential expressions were generated, as shown in Fig. 2, and CFinder software was used to identify modules within the sub-networks. When $\mathrm{k}=4,6$ modules were identified that contained 48 genes. The modules were defined as modules 1-6. (B) DAVID software was used to conduct a GO_BP analysis of each module. GO term enrichment scores were calculated as the $-\log$ (p-value from T test) and were arranged in descending order. The top 10 scores were plotted as a bar chart. (C) DAVID software was also used to perform a pathway analysis of each module. The same method described for (B) was used to graph a bar chart.

research on the 44 determined herbal small molecules. The number of publications was an indicator of the extent of research on the herbal component's use in cancer treatment. In particular, fewer publications were associated with a higher likelihood that the herbal component could become a new anticancer drug. The results revealed that multiple herbal components have been studied extensively and have already been proven to be effective anticancer drugs, such as campto- thecin, staurosporine and resveratrol (the number of relevant publications was $>100$ ). Herbal components, however, have not been extensively studied with respect to their anticancer value, such as lobeline, proscillaridin and cephaeline (the number of relevant publications was $\leq 10$ ), and herbal components, such as helveticoside, hydrastinine, napelline, picrotoxinin and tubocurarine chloride, have never been studied with respect to cancer (Fig. 5). 

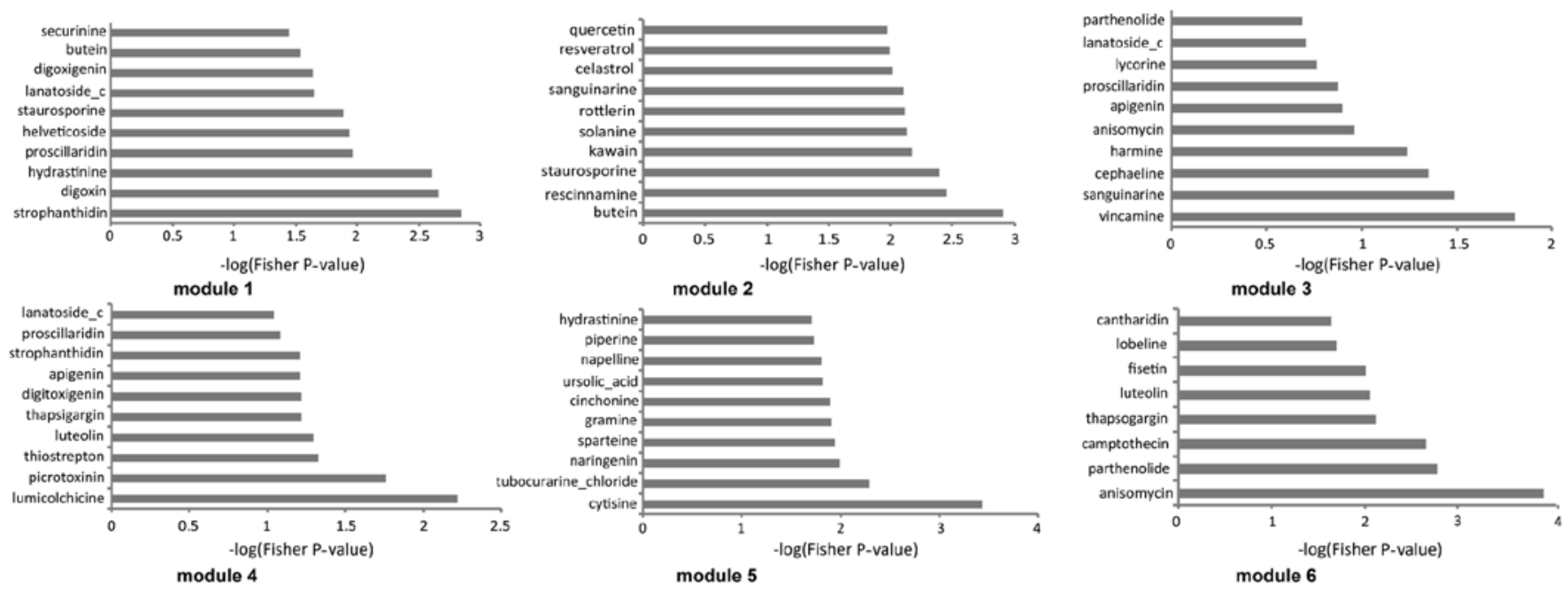

Figure 4. Herbal components that regulate the genes in the 6 core modules were identified through Bioinformatics techniques. Using the cmap database, we calculated that genes were differentially expressed in 1,309 of the small molecule interference experiments examined. The final results showed that 1,221 small molecules caused these differential expressions. Subsequently, the genes from each module were mapped with the differentially expressed genes found from the cmap database, allowing us to determine the relationships between the genes in each module and the tested small molecules. Fisher's exact test was used to calculate the p-value of each herbal component's regulation of each module, and the p-values were converted into statistical scores [statistical score $=-$ log (Fisher p-value)]. The herbal components with the 10 most significant $\mathrm{p}$-values were plotted.

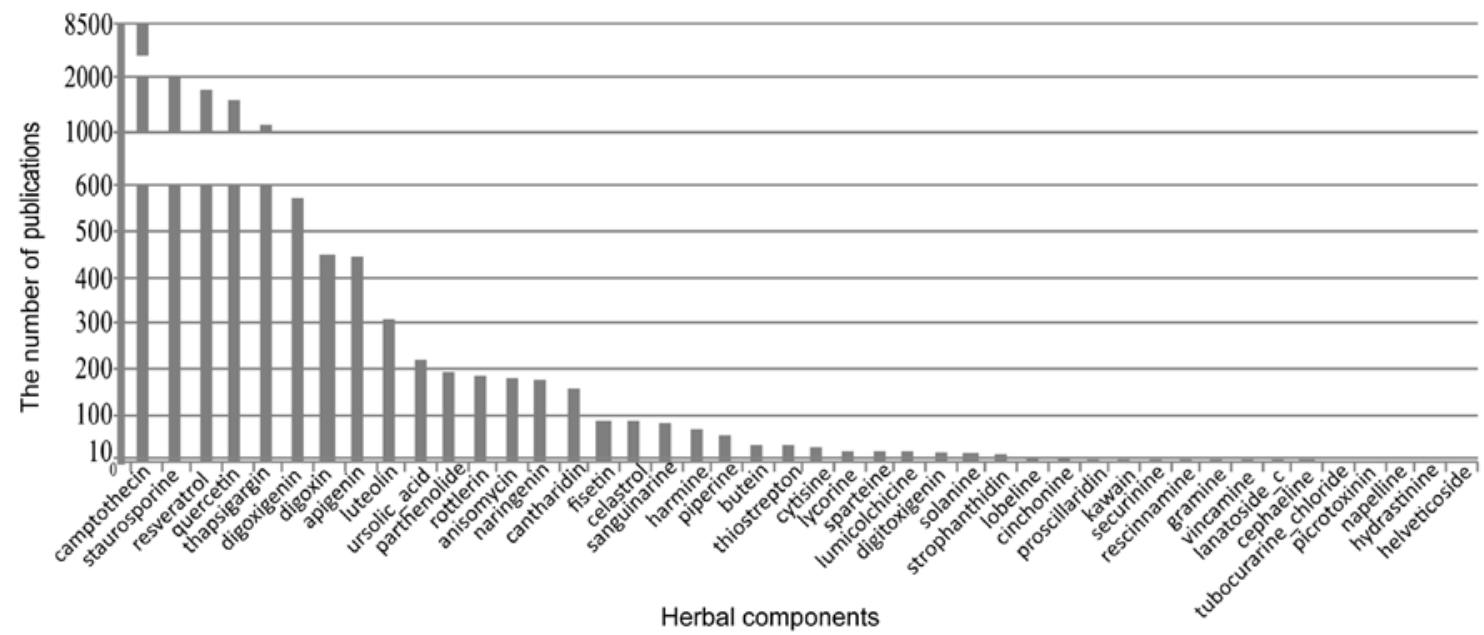

Figure 5. Potential new anticancer drugs that regulate the genes in the 6 core modules were identified through literature review. To obtain novel anticancer drugs, we conducted a literature review of the cancer-related research on the 44 determined herbal small molecules. The number of publications was an indicator of the extent of research on the herbal component's use in cancer treatment. In particular, fewer publications were associated with a higher likelihood that the herbal component could become a new anticancer drug. Fifteen potential new anticancer drugs were identified (the number of relevant publications was $\leq 10$ ).

Creating a regulatory network connecting the herbal components and the modules. Combined with the 44 herbal components and the 6 modules and the results of the literature review, Cytoscape 2.8 was used to generate a regulatory network connecting the herbal components to the modules (Fig. 6).

\section{Discussion}

Normal macrophages exhibit direct anticancer effects, implement innate anticancer immunity and induce the anticancer effects of natural killer (NK) cells and T cells. TAMs, however, are an important part of the tumour microenvironment but exhibit different phenotypes and functions than normal macrophages. Their ability to present antigens is decreased and they can inhibit $\mathrm{T}$ cell proliferation and activity (13). In most solid tumours, macrophages cannot remove the tumour. TAMs even promote tumour development and progression $(14,15)$. Therefore, when developing anticancer therapies, it is essential to obtain an in-depth understanding of the tumour-promoting functions of TAMs and to search for drugs that target and regulate TAM functions, particularly herbs with powerful and wide-ranging anticancer effects.

TAMs have been isolated from a variety of tumour tissues for genome-wide gene chip assays $(16,17)$. In this experiment, we integrated the genome-wide gene chip information already developed for TAMs from different cancer tissue sources, using modular analysis bioinformatics techniques to conduct in-depth analyses of the integrated information. Modular 


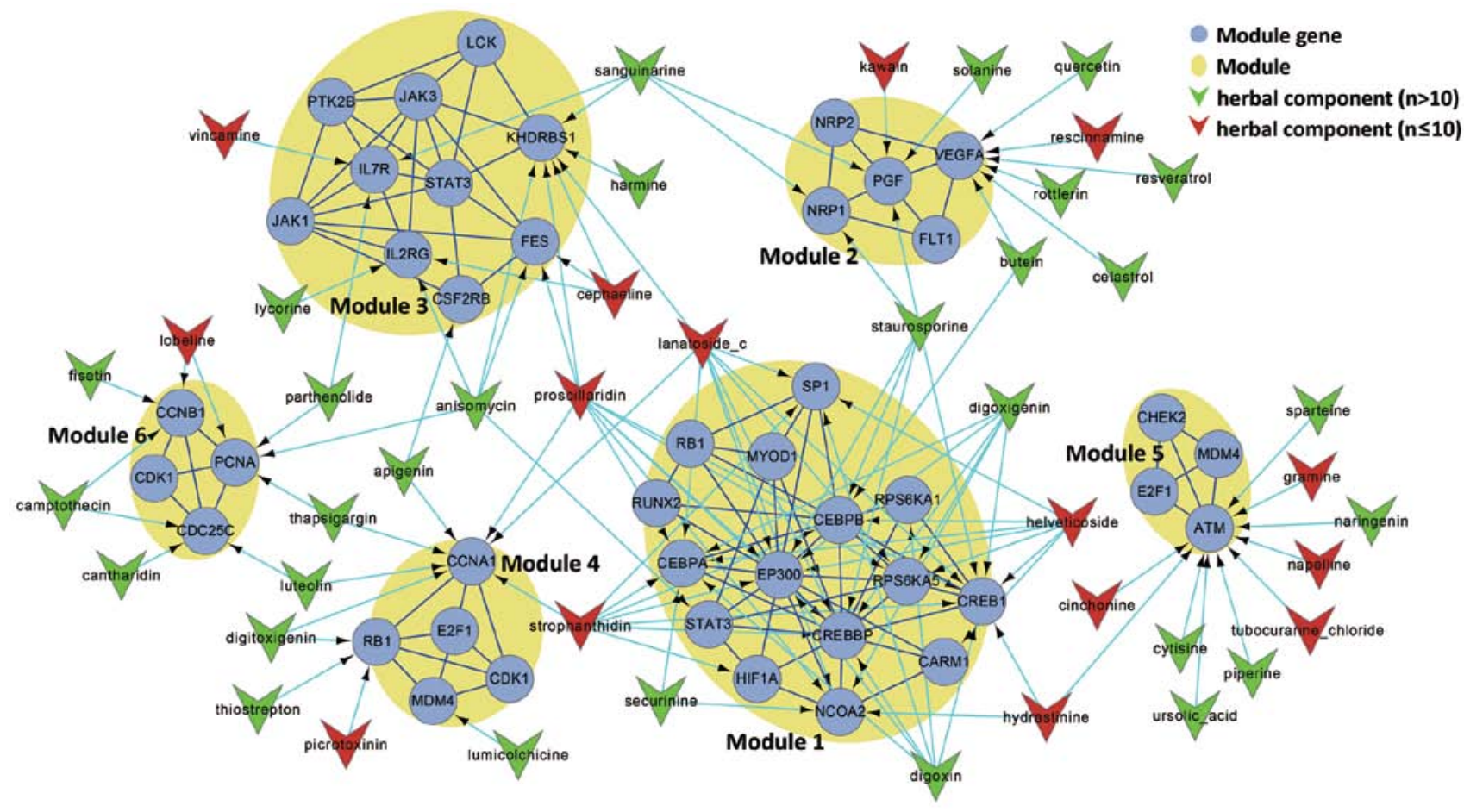

Figure 6. A regulatory network connecting the herbal components and the modules. Combined with the 44 herbal components and the 46 genes in the 6 modules, a regulatory network connecting the herbal components to the modules was generated through Cytoscape 2.8. Polygons represent the herbal components. In particular, red polygons represent herbal components with $\leq 10$ publications and green polygons represent herbal components with $>10$ publications. Blue circles represent the genes in the modules, and blue lines represent gene interactions. Yellow ovals represent the modules themselves, and light green arrows represent the herbal components that regulate the genes in a module.

analysis is a well-established and invaluable bioinformatics technique; it can be used for functional studies of both single proteins and large-scale protein and gene interactions. Moreover, this approach has been used to study the development and treatment of various diseases.

Among the 6 functional modules that underwent modular analysis, module 1 contained the largest number of genes and the most complex interactions among its genes. Module 1 was primarily involved in the positive regulation of gene transcription, expression, biosynthesis and other processes; the expression levels of cancer pathways were also significantly elevated. Among the module 1 genes, the highly expressed gene hypoxia-inducible factor 1A (HIF1A) is a DNA-binding protein that plays an important role in TAM migration. Rapid tumour proliferation can result in a lack of oxygen to tumour cells. This hypoxia may induce the expression of HIF1A-dependent cytokines, which in turn induces the migration of TAMs through the avascular area to the necrotic tissue. Once at the site of necrosis, TAMs play tumour-promoting roles (18). This evidence suggests that module 1 plays an important role in increasing the biological activity of TAMs and regulating their migration. Strong angiogenic ability is a key aspect of tumour cell survival and development, and anti-angiogenic therapy is a popular but difficult objective of anticancer therapies. Studies have demonstrated that TAMs can secrete vascular endothelial growth factor A (VEGFA) and other cytokines into the tumour microenvironment, thereby promoting tumour angiogenesis and development (19). This finding is consistent with the results for module 2 , further demonstrating that the modules screened by modular analysis are responsible for the core functions of TAMs. In module 3, the expression of the JAK-STAT and chemokine signalling pathways, which are closely associated with the development of a variety of tumours, were significantly elevated. Studies have shown that certain tumour-secreted chemokines and interleukins such as chemokine (C-C motif) ligand 2 (CCL2) and interleukin 10 (IL10) can recruit TAMs, increasing the TAM density in the tissue surrounding the tumour and inducing the differentiation of monocytes into TAMs $(2,20)$. These interleukins and chemokines cause this recruitment through the JAK-STAT and chemokine signalling pathways, suggesting that module 3 is involved in mediating the recruitment and migration of TAMs $(21,22)$. Modules 4,5 and 6 were all associated with cell cycle regulation in TAMs. Many experiments and clinical studies have demonstrated that disordered cell cycle regulation is a characteristic of tumours, suggesting that tumour development may result from abnormal cell cycles (23-25). Among the genes in modules 4, 5 and 6, cyclin-dependent kinase $1(\mathrm{CDK} 1)$ and retinoblastoma $1(\mathrm{RB} 1)$ are regarded as the most critical genes for the promotion and inhibition of the cell cycle, respectively $(26,27)$. High CDK1 expression and low RB1 expression are likely critical for the abnormal cell cycles observed in TAMs. The HIF1A, VEGFA, JAK/STAT and CDK1 genes, among others in the last 3 modules, have been shown to be closely associated with cancer development and progression, suggesting that these screened modules contained clusters of key tumour-promoting genes. In each gene group, there were likely synergistic interactions among multiple genes that caused TAMs to play a more powerful 
tumour-promoting role. Thus, in an anticancer therapy, treatment with agents targeting these gene clusters may have better results than treatments targeting only the individual genes.

Traditional Chinese medicine has been used for the treatment of cancer for thousands of years. Chinese herbs have been found to have anticancer effects in both clinical practice and experimental studies. However, few studies have examined the anticancer effects of TAM-targeting herbs. The aim of the present study was to screen for potential anticancer herbal components and provide new theories for targeted cancer treatment research. To that end, we used the cmap small molecule database to screen for herbal components that regulate the core functional modules of TAMs. Among the 44 herbal components found through screening, several components have been extensively studied and already found to have anticancer effects. For instance, the HIF-1 blocker digoxin strongly inhibits tumour growth and metastasis and has been used to treat breast cancer with positive results (28). Camptothecin, however, may disrupt DNA replication and has been shown to repair and treat a variety of cancers, such as breast, uterine and prostate cancer (29). These results indicate that the herbal components we screened do have anticancer effects, confirming the accuracy of the screening.

In addition to the herbal components with proven anticancer effects, our literature review found 15 herbal components upon which few cancer-related studies had been performed. Highly notable are herbal components which have never been addressed in cancer-related studies, such as helveticoside, hydrastinine, napelline, picrotoxinin and tubocurarine chloride. Multiple cardiac glycoside drugs were identified in the 15 herbal components, including lanatosid_c, proscillaridin and helveticoside. There have been reports on cancer treatments using cardiac glycosides from as early as the 1960s; however, due to the toxicity of these compounds to humans, research on these substances was discontinued (10). Studies have noted that the concentration required for cardiac glycosides to induce tumour cell apoptosis does not produce toxicity in humans. Compared with normal tissues, many cancer tissues have altered levels of $\mathrm{Na}^{+} / \mathrm{K}^{+}$-ATPase subunits, and $\mathrm{Na}^{+} / \mathrm{K}^{+}$-ATPase is the target for the inhibitory effect of cardiac glycoside drugs (30-32). The present study found that these molecules could regulate many genes across multiple modules (shown in Fig. 6); for instance, proscillaridin regulated 8 genes in 3 modules, and lanatoside_regulated 9 genes in 3 modules. This evidence suggests that the cardiac glycoside herbal components that have not yet been studied for their anticancer effects have the potential to become new anticancer drugs. As shown in Fig. 6, each module was regulated by at least one of the 15 herbal components, and Fisher's exact test demonstrated that the components' effects were statistically significant. Specifically, strophanthidin was the most significant component regulating module 1. Rescinnamine and kawain were, respectively, the second and fourth most statistically significant components that regulated module 2 . For module 3, vincamine was the most statistically significant component and picrotoxinin was the second. Fisher's exact test results indicated that the 15 herbal components not studied for cancer treatment play a major role in regulating TAM modules, suggesting that these herbs are likely to have strong anticancer effects.
In conclusion, this study integrated TAM experimental results from a variety of cancer tissues and used modular analysis techniques to define 6 modules containing 46 genes associated with TAM's core functions. In addition, based on the cmap database, we found 44 herbal components that can regulate the 6 modules. Fisher's exact test was used to precisely screen for the herbal components that had the most statistically significant module regulation. A literature review was conducted that selected 15 herbal components with considerable potential to become new anticancer drugs, all of which had high statistical significance. Our in-depth and specific analyses of the core functions of TAMs, combined with the screening for herbal components that regulate these TAM modules, provide a new research avenue for future TAM-targeted anticancer therapy. More follow-up studies are required to prove the anticancer effects of the selected herbal components.

\section{Acknowledgements}

This study was supported by the National Natural Science Foundation of China (grant no. 81070373), the National Natural Science Foundation of China for the youth (grant no. 81001081), and the Foundation of the First Affiliated Hospital of Harbin Medical University (grant no. 2011BS018).

\section{References}

1. de Visser KE, Eichten A and Coussens LM: Paradoxical roles of the immune system during cancer development. Nat Rev Cancer 6: 24-37, 2006.

2. Mantovani A, Schioppa T, Porta C, Allavena P and Sica A: Role of tumor-associated macrophages in tumor progression and invasion. Cancer Metastasis Rev 25: 315-322, 2006.

3. Goswami S, Sahai E, Wyckoff JB, et al: Macrophages promote the invasion of breast carcinoma cells via a colony-stimulating factor-1/epidermal growth factor paracrine loop. Cancer Res 65: 5278-5283, 2005

4. Obeid E, Nanda R, Fu YX and Olopade OI: The role of tumorassociated macrophages in breast cancer progression (Review). Int J Oncol 43: 5-12, 2013.

5. Krecicki T, Zalesska-Krecicka M, Jelen M, Szkudlarek T and Horobiowska M: Expression of type IV collagen and matrix metalloproteinase-2 (type IV collagenase) in relation to nodal status in laryngeal cancer. Clin Otolaryngol Allied Sci 26: 469-472, 2001.

6. Lamagna C, Aurrand-Lions M and Imhof BA: Dual role of macrophages in tumor growth and angiogenesis. J Leukoc Biol 80: 705-713, 2006.

7. van Netten JP, Ashmead BJ, Parker RL, et al: Macrophage-tumor cell associations: a factor in metastasis of breast cancer? J Leukoc Biol 54: 360-362, 1993.

8. Terabe M, Matsui S, Park JM, et al: Transforming growth factor- $\beta$ production and myeloid cells are an effector mechanism through which CD1d-restricted T cells block cytotoxic T lymphocytemediated tumor immunosurveillance: abrogation prevents tumor recurrence. J Exp Med 198: 1741-1752, 2003.

9. Wang S, Wu X, Tan M, et al: Fighting fire with fire: poisonous Chinese herbal medicine for cancer therapy.J Ethnopharmacol 140: 33-45, 2012.

10. Newman DJ and Cragg GM: Natural products as sources of new drugs over the last 25 years. J Nat Prod 70: 461-477, 2007.

11. Wang PI and Marcotte EM: It's the machine that matters: predicting gene function and phenotype from protein networks. J Proteomics 73: 2277-2289, 2010.

12. Hedges SB: The origin and evolution of model organisms. Nat Rev Genet 3: 838-849, 2002.

13. Mantovani A, Sozzani S, Locati M, Allavena P and Sica A: Macrophage polarization: tumor-associated macrophages as a paradigm for polarized M2 mononuclear phagocytes. Trends Immunol 23: 549-555, 2002. 
14. Giraudo E, Inoue M and Hanahan D: An amino-bisphosphonate targets MMP-9-expressing macrophages and angiogenesis to impair cervical carcinogenesis. J Clin Invest 114: 623-633, 2004.

15. Mantovani A, Allavena P and Sica A: Tumour-associated macrophages as a prototypic type II polarised phagocyte population: role in tumour progression. Eur J Cancer 40: 1660-1667, 2004.

16. Ojalvo LS, King W, Cox D and Pollard JW: High-density gene expression analysis of tumor-associated macrophages from mouse mammary tumors. Am J Pathol 174: 1048-1064, 2009.

17. Zabuawala T, Taffany DA, Sharma SM, et al: An Ets2-driven transcriptional program in tumor-associated macrophages promotes tumor metastasis. Cancer Res 70: 1323-1333, 2010.

18. Sica A, Schioppa T, Mantovani A and Allavena P: Tumourassociated macrophages are a distinct M2 polarised population promoting tumour progression: potential targets of anti-cancer therapy. Eur J Cancer 42: 717-727, 2006.

19. Lewis GE, Leek R, Harris A and McGee JO: Cytokine regulation of angiogenesis in breast cancer: the role of tumor-associated macrophages. J Leukoc Biol 57: 747-751, 1995.

20. Espey MG: Tumor macrophage redox and effector mechanisms associated with hypoxia. Free Radic Biol Med 41: 1621-1628, 2006.

21. Riley JK, Takeda K, Akira S and Schreiber RD: Interleukin-10 receptor signaling through the JAK-STAT pathway. Requirement for two distinct receptor-derived signals for anti-inflammatory action. J Biol Chem 274: 16513-16521, 1999.

22. Ksiazkiewicz M, Gottfried E, Kreutz M, Mack M, Hofstaedter F and Kunz-Schughart LA: Importance of CCL2-CCR2A/2B signaling for monocyte migration into spheroids of breast cancerderived fibroblasts. Immunobiology 215: 737-747, 2010.

23. Gao YF, Li T, Chang Y, et al: Cdk1-phosphorylated CUEDC2 promotes spindle checkpoint inactivation and chromosomal instability. Nat Cell Biol 13: 924-933, 2011.
24. Kastan MB and Bartek J: Cell-cycle checkpoints and cancer. Nature 432: 316-323, 2004.

25. Vermeulen K, Van Bockstaele DR and Berneman ZN: The cell cycle: a review of regulation, deregulation and therapeutic targets in cancer. Cell Prolif 36: 131-149, 2003.

26. Gravells P, Tomita K, Booth A, Poznansky J and Porter AC: Chemical genetic analyses of quantitative changes in $\mathrm{Cdk} 1$ activity during the human cell cycle. Hum Mol Genet 22: 2842-2851, 2013.

27. Nemtsova MV, Zemliakova VV, Kuznetsova EV, et al: Analysis of the correlation between genetic and immunohistochemical markers (RB1, p16 and p53 gene regultors of a cell cycle) in sporadic mammary gland carcinoma. Arkh Patol 69: 3-6, 2007 (In Russian).

28. Wong CC, Zhang H, Gilkes DM, et al: Inhibitors of hypoxiainducible factor 1 block breast cancer metastatic niche formation and lung metastasis. J Mol Med 90: 803-815, 2012.

29. Beretta GL, Gatti L, Perego P and Zaffaroni N: Camptothecin resistance in cancer: insights into the molecular mechanisms of a DNA-damaging drug. Curr Med Chem 20: 1541-1565, 2013.

30. Kolkhof P, Geerts A, Schäfer S and Torzewski J: Cardiac glycosides potently inhibit C-reactive protein synthesis in human hepatocytes. Biochem Biophys Res Commun 394: 233-239, 2010

31. Schoner W and Scheiner-Bobis G: Endogenous and exogenous cardiac glycosides: their roles in hypertension, salt metabolism, and cell growth. Am J Physiol Cell Physiol 293: C509-C536, 2007.

32. Bagrov AY and Shapiro JI: Endogenous digitalis: pathophysiologic roles and therapeutic applications. Nat Clin Pract Nephrol 4: 378-392, 2008. 